\title{
On the Continuity of the Critical Value for Long Range Percolation in the Exponential Case
}

\author{
Ronald Meester ${ }^{1}$, Jeffrey E. Steif ${ }^{2}$ \\ ${ }^{\prime}$ University of Utıecht, Department of Mathematics, P O Box 80010,3508 TA Utrecht, The \\ Netherlands E-ma1l: mecster@math ruunl \\ 2 Department of Mathematics, Chalmers University of Technology, S-412 96 Gothenburg, Sweden \\ E-mail steif(u) math chalmers se
}

Received: 28 December 1994/Accepted: 4 March 1996

\begin{abstract}
We show that for a long range percolation model with exponentially decaying connections, the limit of critical values of any sequence of long range percolation models approaching the original model from below is the critical value for the original long range percolation model As an interesting corollary, this implies that if a long range percolation model with exponential connections is supercritical, then it still percolates even if all long bonds are removed We also show that the percolation probability is continuous (in a certain sense) in the supercritical regime for long range percolation models with exponential connections.
\end{abstract}

\section{Introduction}

The purpose of this paper is to investigate the continuity from below of the critical value for long range percolation models We begin with a description of long range percolation We let $\mathbf{Z}^{d}$ denote the standard $d$-dimensional cubic lattice with the usual $L_{1}$ norm given by $\left|\left(x_{1}, x_{2}, \quad, x_{d}\right)\right|=\sum_{l=1}^{d}\left|x_{i}\right|$ If $S$ and $T$ are subsets of $\mathbf{Z}^{d}$, we let $d(S, T)=\min _{s \in S, t \in T}|s-t|$ be the $L_{1}$ distance between $S$ and $T$. If $|x-y|=1$, we call $r$ and $y$ nearest neighbors We will also sometimes need the $L_{\infty}$ norm given by $\left\|\left(x_{1}, x_{2}, ., x_{d}\right)\right\|=\max _{l}\left|x_{l}\right|$ Everything we will define in this paper will implicitly depend on the dimension $d$ However, we will not mention this $d$, and all our results will be valid for any $d \geqq 2$. Given a set $R \subseteq \mathbf{Z}^{d}$, we let $\Delta^{l}(R)=\{x \notin R$ $|x-y|=1$ for some $y \in R\}$ and call this the vertex boundary of $R$ We also let $U^{e}(R)=\{\{x, y\} \quad x \in R, y \notin R,|x-y|=1\}$ and call this the edge boundary of $R$

We now introduce probability into the setup For $n=1,2$, let $p_{n} \in[0,1)$ be such that

$$
\sum_{0 \neq r \in \mathbf{Z}^{\prime l}} p_{|\lambda|}<\infty
$$

We picture edges or bonds $\{x, y\}$ between all pairs $x$ and $y, x \neq y$, and declare such an edge to be open with probability $p_{n}$ if $|x-y|=n$ independently of all

\footnotetext{
* Research supported by a grant from the Swedish National Science Foundation
} 
other edges (Formally an edge is a subset of $\mathbf{Z}^{d}$ with two elements and the length of an edge will be the $L_{1}$ distance between the two points comprising the edge.) By Borel-Cantelli, the convergence of the above sum is equivalent to every vertex having a finite number of edges emanating from it a $\mathrm{s}$

We will sometimes write $p(x)$ by which we will mean $p_{|\uparrow|}$ An open path is a sequence of vertices $x_{0}, x_{1}, ., x_{n}$, where $\left\{x_{l}, x_{l+1}\right\}$ is open for $i=0,, n-1$ Let the origin be denoted by 0 and let

$$
C=\left\{x \in \mathbf{Z}^{d} \quad \exists \text { an open path from } 0 \text { to } x\right\},
$$

which we call the cluster of the origin and we let

$$
0\left(p_{1}, p_{2}, \quad\right)=P(|C|=\infty),
$$

which we call the percolation function, which depends on $p_{1}, p_{2}, \quad$ (as well as on d) If $\theta\left(p_{1}, p_{2}, \quad\right)>0$, we say that there is percolation under $p_{1}, p_{2}$,

An interesting and historically important case is when $p_{l}=0$ for $i \geqq 2$ The above percolation function then simply becomes a function of one variable, which we write as $\theta^{(d)}(p)$. In this case, it had been known for a long time that for $d \geqq 2$, $o^{(d)}(p)$ is 0 for $p$ near 0 and positive for $p$ near 1 , the latter already making things interesting In two dimensions, it was proved already in 1960 (see [7]) that $0^{(2)}(1 / 2)=0$ Building upon work of Russo, Seymour and Welsh, Kesten (see [8]) proved that $\theta^{(2)}(p)>0$ for $p>1 / 2$ Combining these two statements, we write $p_{c}(2)=1 / 2$ and say that the critical value in two dimensions is $1 / 2$ for ordinary bond percolation, ordinary meaning that $p_{2}=p_{3}==0$

The usual approach to long range percolation in one dimension (see [9] and [2]) is that one fixes all the connection probabilities $p_{2}, p_{3}$, and then considers $p_{1}$ as a parameter We will use this approach as well in which case it is then natural to define the critical probability $p_{c}\left(p_{2}, p_{3}, \quad\right)$ as

$$
p_{c}\left(p_{2}, p_{3}, \quad\right)=\inf \left\{p_{1} \quad O\left(p_{1}, p_{2}, \ldots\right)>0\right\},
$$

noting of course that this depends on $p_{2}, p_{3}$, (as well as on $d$ ) It is natural to think of the cases $p_{1}>p_{c}\left(p_{2}, p_{3}, \quad\right), p_{1}<p_{c}\left(p_{2}, p_{3},.\right)$ and $p_{1}=$ $p_{c}\left(p_{2}, p_{3}, \quad\right)$ as the supercritical, subcritical, and critical regimes respectively.

The main result of this paper is a sufficient condition under which the critical value is continuous from below for long range percolation models To state this result, we first state a condition which we will assume the model satisfies This condition does not seem natural but we will see afterwards that models with exponentially decaying connections satisfy it. Before stating the condition, we need to introduce some notation which comes from [6]

For each positive integer $n$, we let $B(n)$ be the set

$$
\left\{x \in \mathbf{Z}^{d} \quad\|x\| \leqq n\right\}
$$

We say that $p_{2}, p_{3}, \quad$ satisfies Condition $\mathrm{C}$ if for all $p_{1} \in(0,1)$, there exists $c>0$ such that for all $n \geqq 1$, if $R \subseteq B(n), \gamma \in B(n)$ and $\gamma \notin R \cup \Delta^{l}(R)$, then

$$
\sum_{i \in \Delta^{\prime}(R) \cap B(n)} p\left(x-y^{\prime}\right) \geqq c \sum_{i \in\left(R \cup d^{\prime}(R)\right) \cap B(n)} p\left(x-\eta^{\prime}\right) .
$$


Remarks. 1 Note that this inequality implies that if $\gamma \notin R \cup \Delta^{\prime}(R)$, then

$$
\sum_{r \in A^{\prime}(R)} p\left(x-\hat{\imath}^{\prime}\right) \geqq c \sum_{i \in R \cup \Delta^{\prime}(R)} p(x-\hat{\imath})
$$

This is clear if $R$ is finite since we can then simply take $n$ large enough in Condition $C$ Next, once we know this for finite $R$, a simple limiting argument proves this for all $R$ (using the fact that $\sum_{0 \neq r \in \mathbf{Z}^{\prime}} p_{|\hat{\gamma}|}<\infty$ )

2 The reader can check that if for some $p_{1} \in(0,1)$, there exists a $c$ with the desired property, then the same is true for all $p_{1} \in(0,1)$ (although the constant $c$ will of course depend on $p_{1}$ )

3 Similarly, the reader can check that assuming $p_{i}=0$ implies that $p_{i+1}=0$, then whether $p_{2}, p_{3}$, satisfies Condition $\mathrm{C}$ only depends on the tail of the sequence

Theorem 1.1. Let $d \geqq 2$ Assume that $p_{2}, p_{3}$, satisfies Condition $\mathrm{C}$, and that $p_{2}^{n}, p_{3}^{n}, \quad$ are such that for all $i \geqq 2$ and $n \geqq 1, p_{i}^{n} \leqq p_{i}$ and that for all $i \geqq 2$, $\lim _{n \rightarrow \infty} p_{l}^{n}=p_{l}$ Then

$$
\lim _{n \rightarrow \infty} p_{c}\left(p_{2}^{n}, p_{3}^{n}, \quad\right)=p_{c}\left(p_{2}, p_{3}, \quad\right) .
$$

As an immediate corollary, we obtain the fact that a supercritical long range model which satisfies Condition $C$ percolates if we cut off all long edges

Corollary 1.2. Let $d \geqq 2$ Assume that $p_{2}, p_{3}$, satisfies Condition $\mathrm{C}$ and let $p_{1}>p_{c}\left(p_{2}, p_{3}, \quad\right)$ Then there exists some integer $k$ so that there is percolation under $\left(p_{1}, p_{2}, \quad, p_{h}, 0,0, \quad\right)$

It is easy to show that in 1 dimension, if $p_{2}, p_{3}$, . satisfies Condition $\mathrm{C}$ and $p_{1}<1$, then the model does not percolate It follows that Theorem 11 is then also true in 1 dimension since all the critical values involved are 1 However, in 1 dimension, Theorem 11 cannot be true in general with the assumption of Condition $\mathrm{C}$ removed, since in 1 dimension, long range percolation models can percolate (see [9] and [2]) while finite range models cannot

The next result tells us that a long range model with exponential connections satisfies Condition $\mathrm{C}$. We do not believe that Condition $\mathrm{C}$ is much stronger than having exponential connections but we find it convenient to have stated our results in this way

Theorem 1.3. Let $d \geqq 2$ Assume that $\left\{p_{n}\right\}_{n=2}^{\infty}$ is such that either

(i) the sequence $\left\{p_{n}\right\}$ is strictly positive up to a finite point and then becomes identically, 0 after that, or

(ii) the sequence $\left\{p_{n}\right\}$ is strictly positive and eventually exponential, meaning that there exist $\alpha>0, \rho \in(0,1)$ and $N_{0} \geqq 2$ so that $p_{n}=\alpha \rho^{n}$ for $n \geqq N_{0}$ Then $\left\{p_{n}\right\}_{n=2}^{\infty}$ satisfies Condition $\mathrm{C}$

We next mention an application of Theorem 11 whose proof uses a method developed in [3] It says that if a sequence converges in $L_{1}$ to a supercritical model which satisfies Condition $\mathrm{C}$, then the percolation probabilities converge to that of the limit It is also the case that if the sequence converges in $L_{1}$ from above, then this is still true without either the supercriticality or Condition $C$ assumption 
We note that any long range model $p_{1}, p_{2}$, can be viewed as a real valued function on $\mathbf{Z}^{d}$ by $p_{1}, p_{2}, \quad .(x)=p_{|\wedge|}$

Theorem 1.4. Let $d \geqq 2$ Assume that $p_{1}^{n}, p_{2}^{n}, p_{3}^{n}$, converges to $p_{1}, p_{2}, p_{3}$, in $L_{1}$ of $\mathbf{Z}^{d}$ with counting measure (and hence pointwise) Assume also that for all $i \geqq 1$ and $n \geqq 1, p_{l}^{n} \geqq p_{i}$ Then

$$
\lim _{n \rightarrow \infty} \theta\left(p_{1}^{n}, p_{2}^{n}, .\right)=0\left(p_{1}, p_{2}, \quad .\right)
$$

Assume that $p_{2}, p_{3}, \quad$ satisfies Condition $\mathrm{C}$ and that $p_{1}>p_{c}\left(p_{2}, p_{3}, \quad\right)$ Then for all sequences $p_{1}^{n}, p_{2}^{n}, p_{3}^{n}$, which converge to $p_{1}, p_{2}, p_{3}$, in $L_{1}$ of $\mathbf{Z}^{\text {d with }}$ counting measure (and hence pointwise), it follows that

$$
\lim _{n \rightarrow \infty} \theta\left(p_{1}^{n}, p_{2}^{n}, \quad\right)=0\left(p_{1}, p_{2}, .\right)
$$

We end this section with a rough sketch of the mechanism used to prove Theorem 11 Actually, it is a little clearer to concentrate on Corollary 1.2 at this point The approach is based on the renormalization technique of Grimmett and Marstrand ([6]) Consider a rectangular grid of large disjoint cubes with side length $N$, say We order these boxes in some way and investigate each of these boxes in the given order, declaring each of these boxes to be either occupied or vacant according to some rule Three things will be important for this rule

(i) the probability for a new box to be occupied, conditioned on the past of the procedure, should always be larger than, and uniformly bounded away from, the critical probability for ordinary nearest neighbor independent site percolation,

(ii) the rule by which we declare boxes to be occupied or vacant should be compatible with the underlying long range percolation in the sense that if there is a nearest neighbor path of occupied boxes, then there must be a corresponding open path in the underlying model, visiting all boxes in this open path,

(iii) the event that a certain box is occupied should depend on the state of a uniformly bounded number of edges with a uniformly bounded length

The strategy is to show that if there is percolation in a certain long range model, then we can find some large $N$ and an appropriate rule with the properties mentioned above In such a case, it follows that the renormalized model percolates as a nearest neighbor model, which implies, using properties (ii) and (iii), that the underlying model percolates using only edges up to a certain finite length It is clear that one of the key steps is to define a suitable rule

The outline of the present paper is as follows In Sect 2, we will prove the main lemma needed to obtain Theorem 11 This lemma is a long range analogue of Lemma 26 in [6] Once this lemma is proved, we carry out in Sect 3 the renormalization and show how it leads to a proof of Theorem 11 In Sect 4, we will prove Theorem 14 as well as a number of other applications of Theorem 1.1 Finally, the proof of Theorem 13, which is intuitively obvious, will be given in Sect 5

We end this section by mentioning the paper [1] which also contains results concerning continuity (and strict monotonicity) of the critical value of certain models Some of the systems considered there are certain percolation models and ferromagnetic Ising models 


\section{The Main Lemma}

We begin this section with a certain amount of notation, and we will then state Lemma A, the main result of this section With only this notation and Lemma A, the reader can continue on to Sect 3 to read the proof of Theorem 1.1 After the statement of Lemma A, this section will be devoted exclusively to its proof and will not be needed otherwise

If $E$ is a set of edges and $V$ is a set of vertices, we say $E$ is contained in $V$ ( $E \subseteq V$ ) if both endpoints (or elements) of all edges in $E$ are contained in $V$ We also let $E \cap V$ denote the set of edges in $E$ both of whose endpoints are in $V$ If $A$ and $B$ are disjoint subsets of $\mathbf{Z}^{d}$, we write $A \leftrightarrow B$ if there is an open path from some point of $A$ to some point of $B$ If $A$ and $B$ are as above with $A \cup B \subseteq C$, then $A \leftrightarrow B($ in $C$ ) will mean that there is an open path contained in $C$ from some point of $A$ to some point in $B$ Finally, we will allow ourselves to write $A \leftrightarrow B($ in $C$ ) even if $A$ and/or $B$ are not subsets of $C$ by which we will mean that the open path between $A$ and $B$ is contained in $C$ as much as possible in the obvious sense For example, if $A \subseteq C$ but $B \nsubseteq C$, then all vertices of the path are required to be in $C$ except for the last one. We also write $A \rightarrow \infty$ when there is an open path from some point of $A$ to $\infty$

We introduce two further subsets of $\mathbf{Z}^{d}$ Let $T(n)$ be the set

$$
\left\{x=\left(x_{1}, x_{2}, \quad, x_{d}\right) \in \mathbf{Z}^{d} \cdot x_{1}=n, 0 \leqq x_{j} \leqq n \quad \text { for } j=2, \quad, d\right\},
$$

which is a special subset of a face of $B(n)$ For positive integers $m$ and $n$ with $2 m<n$, we also let

$$
T(m, n)=\bigcup_{j=1}^{2 m+1}\left\{j e_{1}+T(n)\right\},
$$

where $e_{j}$ is the vector which is 1 in the $j^{\text {th }}$ coordinate and 0 otherwise

Next, a translate of $B(m)$ is called an open $m$-pad if each pair of vertices in this set is connected by an open path in this set Finally, we let $K(m, n)$ be the subset of $T(n)$ consisting of all vertices $x$ which are such that there is an open edge from $x$ to $x+e_{1}$ and $x+e_{1}$ is contained in an open $m$-pad which is contained in $T(m, n)$

It will turn out to be useful to introduce continuous uniform random variables for the edges of length 1 which will allow us to simultaneously couple all of the processes as $p_{1}$ varies and with $p_{l}$ for $i \geqq 2$ fixed. Let $E_{1}$ denote all edges of length 1 (or equivalently all subsets of 2 elements consisting of nearest neighbors), let $E_{2}$ denote all edges of length larger than 1 and let $\Omega=[0,1]^{E_{1}} \times\{0,1\}^{E_{2}}$ equipped with the usual $\sigma$-algebra and with product measure $P$ where each measure on $[0,1]$ is uniform, and if $e \in E_{2}$ has length $k(\geqq 2)$ then the marginal for $e$ is $p_{k} \delta_{1}+(1-$ $\left.p_{k}\right) \delta_{0}$ (Of course, for $e \in E_{2}, 1$ represents the edge being open.) For $x \in E_{1}$ and (1) $\in \Omega$, we let $\omega(x)$ denote the value at $x$ (which has a uniform distribution) We say that $x \in E_{1}$ is $\rho$-open if $\omega(x)<\rho$ and that it is $\rho$-closed if $\omega(x) \geqq \rho$. If we look at the set of $\rho$-open edges of length 1 together with the open edges of length larger than 1, we obtain a realization of long range percolation with parameters $\rho, p_{2}, p_{3}$,

We can now state the main result of this section.

Lemma A. Let $p_{2}, p_{3}$, satisfy, Condition $\mathrm{C}, p \in(0,1)$ and $0\left(p, p_{2}, p_{3}, \quad\right)>0$ and let $\varepsilon, \delta>0$ Then there exists $m$ and $n$ such that $2 m<n$ and such that 
if $B(m) \subseteq R \subseteq B(n),\left(R \cup \Delta^{\prime}(R)\right) \cap T(n)=\emptyset$ and if $\beta . \Delta^{e}(R) \cap B(n) \rightarrow[0,1-\delta]$, then $P(G \mid H)>1-\varepsilon$, where

$$
H=\left\{\forall e \in \Delta^{e}(R) \cap B(n), e \text { is } \beta(e) \text {-closed }\right\}
$$

and $G$ is the event that there is a path ' from $R$ to $K(m, n)$ in $B(n)$, such that only, the first vertex is in $R$, the first edge $e$ is of length 1 and is $(\beta(e)+\delta)$-open, and all subsequent edges are open, the edges of length 1 after the first being p-open

For the rest of this section, we have a fixed $p_{2}, p_{3}$. satisfying Condition $\mathrm{C}$ and a $p \in(0,1)$ such that $\theta\left(p, p_{2}, \quad\right)>0$ There therefore exists $c>0$ such that for all $n \geqq 1$, if $R \subseteq B(n), ; \in B(n)$ and $; \in R \cup \Delta^{\prime}(R)$, then

$$
\sum_{r \in \Delta^{\prime}(R) \cap B(n)} p\left(x-\gamma^{\prime}\right) \geqq c \sum_{i \in\left(R \cup \Delta^{\prime}(R)\right) \cap B(n)} p\left(x-\gamma^{\prime}\right)
$$

(where $p(y)=p$ if $|y|=1$ )

Remark From a previous remark, this inequality implies that if $R \subseteq \mathbf{Z}^{d}$ and $\gamma \notin R \cup A^{\prime}(R)$, then

$$
\sum_{r \in \Delta^{\imath}(R)} p\left(x-\gamma^{\prime}\right) \geqq c \sum_{i \in R \cup \Delta^{t}(R)} p\left(x-\gamma^{\prime}\right)
$$

We start off with three elementary lemmas concerning independent 0,1 -valued random variables

Lemma 2.1. Let $\tilde{c}>0$ Let $\left\{X_{i}\right\}_{i \in I}$ be independent 0,1 valued random variables with $p_{l}=P\left(X_{l}=1\right)$ Let $\left\{Y_{j}\right\}_{l \in J}$ be independent 0,1 valued random variables with $p_{I}=P\left(Y_{I}=1\right)$ If $\sum_{i \in I} p_{i} \geqq \tilde{c} \sum_{J \in J} p_{j}$ then for all integers $k$ and $\varepsilon>0$, either

$$
P\left(\sum_{i \in I} X_{l} \leqq k\right) \leqq \varepsilon
$$

or

$$
P\left(\sum_{j \in J} Y_{J} \geqq \frac{4}{\tilde{c} \varepsilon^{2}}+\frac{2 k}{\tilde{c} \varepsilon}\right) \leqq \varepsilon
$$

Proof If the second inequality fails, then $E\left[\sum_{j \in J} Y_{J}\right] \geqq \frac{4}{\bar{c} \varepsilon}+\frac{2 k}{\bar{c}}$ It follows by our assumption that $E\left[\sum_{i \in I} X_{i}\right] \geqq \frac{4}{c}+2 k$, whence

$$
\begin{aligned}
P\left(\sum_{i \in I} X_{l} \leqq k\right) & \leqq P\left(\left|\sum_{i \in I} X_{l}-E\left[\sum_{i \in I} X_{l}\right]\right| \geqq E\left[\sum_{i \in I} X_{l}\right]-k\right) \\
& \leqq \frac{\operatorname{Var}\left[\sum_{i \in I} X_{i}\right]}{\left(E\left[\sum_{i \in I} X_{l}\right]-k\right)^{2}} \leqq \frac{E\left[\sum_{l \in I} X_{l}\right]}{\left(E\left[\sum_{i \in I} X_{l}\right]-k\right)^{2}} \\
& \leqq \frac{E\left[\sum_{i \in I} X_{l}\right]}{\left(\frac{l\left[\sum_{i \in I} l_{l}\right]}{2}\right)^{2}}=\frac{4}{E\left[\sum_{i \in I} X_{l}\right]} \leqq \varepsilon
\end{aligned}
$$

The first and fourth inequalities follow from the fact that $E\left[\sum_{i \in I} X_{i}\right] \geqq 2 k$ and the second inequality is Chebyshev's inequality 
Lemma 2.2. Given $M$ and 8 there exists $L=L(M, \varepsilon)$ such that if $X_{1}, X_{2}, \ldots$ are independent 0,1 valued random variables with $p_{l}=P\left(X_{i}=1\right)$ and $\sum_{i} p_{l} \leqq M$, then for all subsets $S \subseteq\{1,2, \quad\}$, we have

$$
P\left(\sum_{i=1}^{\infty} X_{l} \geqq L \mid \sum_{l \in S} X_{l} \geqq 1\right)<\varepsilon
$$

Proof We can assume without loss of generality that $p_{i}>0$ for all $i \in S$ (otherwise leave out the indices $i$ for which $p_{l}=0$, the event on which we condition remains the same) Conditioned on $\sum_{l \in S} X_{l} \geqq 1$, the distribution of $X_{i}, i \notin S$ is unchanged and for $i \in S, X_{l}=1$ with probability

$$
\frac{p_{l}}{1-\prod_{, \in S}\left(1-p_{i}\right)}
$$

We next show that the sum of the new $p_{l}$ 's (conditioned on $\sum_{l \in S} X_{l} \geqq 1$ ) is bounded, uniformly in $S$ To do this, we need only consider the sum over $S$ which is done as follows

$$
\frac{\sum_{i \in S} p_{l}}{1-\prod_{i \in S}\left(1-p_{j}\right)} \leqq \frac{\sum_{l \in S} p_{i}}{1-\exp \left(-\sum_{j \in S} p_{l}\right)} \leqq \sup _{i \in(0 M]} \frac{x}{1-\exp (-x)}<x
$$

Finally an application of Markov's inequality completes the proof.

Lemma 2.3. Given $b, \gamma \in(0,1)$ and $N \geqq 1$, there exists $\delta=\delta(b, \gamma, N)$ such that if $\left\{X_{i}\right\}_{l} \in l$ are independent Bernoulli random rariables with $p_{l}=P\left(X_{i}=1\right) \leqq b$ for all $i$ and such that $P\left(\sum_{i \in I} X_{i} \geqq 1\right) \geqq 1-\delta$, then $P\left(\sum_{i \in I} X_{i} \geqq N\right) \geqq 1-$;

Proof Choose $\alpha=\alpha(b)>0$ so that $1-x \geqq e^{-x}$ for all $x \in[0, b]$ Let $\delta$ be such that $-\log \delta / \alpha>2 N$ and $4 \alpha /-\log \delta<i$ Then, a simple calculation shows that $P\left(\sum_{l \in I} X_{l} \geqq 1\right) \geqq 1-\delta$ implies that $\sum_{i \in I} p_{l} \geqq-\log \delta / \alpha$ which is in turn larger than $2 N$ Chebyshev's inequality then gives

$$
\begin{aligned}
P\left(\sum_{i \in I} X_{l}<N\right) & \leqq P\left(\left|\sum_{i \in I} X_{l}-\sum_{i \in I} p_{i}\right| \geqq \frac{\sum_{l \in I} p_{l}}{2}\right) \\
& \leqq \frac{\operatorname{Var}\left(\sum_{l \in I} X_{l}\right)}{\left(\frac{\sum_{i \in I} p_{i}}{2}\right)^{2}} \leqq \frac{4}{\sum_{l \in I} p_{i}} \leqq \frac{4 \alpha}{-\log \delta}<{ }^{\prime}
\end{aligned}
$$

Lemmas 24 and 25 below are the key ideas we use to tame the fact that we have long range percolation rather than finite range percolation Lemma 24 tells us that if there are many open edges from a certain random set to a fixed set together with its vertex boundary, then there are a fair number of open edges from this random set to the vertex boundary of the fixed set with high probability Lemma 25 in turn tells us that if there are many open edges from a certain random set to a fixed set, then there are a fair number of vertices in the fixed set with an open edge to this random set with high probability (Note that for finite range models, or more generally, when there is an a priori bound on the degree of a vertex, this is obvious ) 
Lemma 2.4. Let $R \subseteq B(n)$ with $\left(R \cup A^{l}(R)\right) \cap T(n)=\emptyset$ Let $S$ be a random subset of $\left(R \cup \Delta^{\prime \prime}(R)\right)^{c} \cap B(n)$ which is measurable with respect to the edges contained in $\left(R \cup A^{\prime \prime}(R)\right)^{c}$ Let $E$ be the set of open edges from $S$ to $\Delta^{\prime}(R) \cap B(n)$ and let $F$ be the set of open edges from $S$ to $\left(R \cup \Delta^{\prime}(R)\right) \cap B(n)$ Then for all integers $k$ and all $:>0$,

$$
P\left(|E| \leqq k,|F| \geqq \frac{4}{c \varepsilon^{2}}+\frac{2 k}{c \varepsilon}\right) \leqq \varepsilon,
$$

where $c$ is as in (2 1) Also, if $R \subseteq \mathbf{Z}^{d}, S$ is a random subset of $\left(R \cup \Delta^{r}(R)\right)^{c}$ which is measurable with respect to the edges contained in $\left(R \cup \Delta^{l}(R)\right)^{c}, E$ is the set of open edges from $S$ to $A^{l}(R)$ and $F$ is the set of open edges from $S$ to $R \cup \Delta^{v}(R)$, then for all integers $k$ and all $\varepsilon>0$,

$$
P\left(|E| \leqq k,|F| \geqq \frac{4}{c \varepsilon^{2}}+\frac{2 k}{c \varepsilon}\right) \leqq \varepsilon
$$

Proof We prove only the first case where $R \subseteq B(n)$ with $\left(R \cup \Delta^{l}(R)\right) \cap T(n)=\emptyset$ The second statement is proved identically using the remark after (21). By independence of different edges, it suffices to show that

$$
P\left(|E| \leqq k,|F| \geqq \frac{4}{c \varepsilon^{2}}+\frac{2 k}{c \varepsilon} \mid S=\Gamma\right) \leqq \varepsilon
$$

for all $\Gamma \subseteq\left(R \cup \Lambda^{l}(R)\right)^{c} \cap B(n)$ with $P(S=\Gamma)>0$. To do this, it suffices by the FKG inequality (see [5]) and the assumed measurability of $S$ (with respect to the edges outside of $\left.R \cup \Delta^{l}(R)\right)$ to show

$$
P(|E| \leqq k \mid S=\Gamma) \quad P\left(|F| \geqq \frac{4}{c \varepsilon^{2}}+\frac{2 k}{c \varepsilon} \mid S=\Gamma\right) \leqq \varepsilon
$$

for all $\Gamma \subseteq\left(R \cup \Delta^{l}(R)\right)^{c} \cap B(n)$ with $P(S=\Gamma)>0$ Fix such a $\Gamma$ By Lemma 21 , it suffices to show that

$$
\sum_{i \in A^{\prime}(R) \cap B(n), ; \in I} p\left(x-i^{\prime}\right) \geqq c \sum_{r \in\left(R \cup A^{\prime}(R)\right) \cap B(n), ; \in I} p(x-i)
$$

By summing over $i \in I$ last, this follows from (2 1)

Lemma 2.5. Given s and $k$, there exists a $(k, \varepsilon)$ such that if $S_{1} \subseteq \mathbf{Z}^{d}, S_{2}$ is a random subset of $\mathbf{Z}^{d} \backslash S_{1}$ which is measurable with respect to the edges contained in $\mathbf{Z}^{d} \backslash S_{1}$, $E$ is the set of open edges from $S_{2}$ to $S_{1}$, and $V$ is the set of vertices in $S_{1}$ which are an endpoint of an open edge to $S_{2}$, then

$$
P(|V| \leqq k,|E| \geqq a(k, \varepsilon)) \leqq \varepsilon
$$

Proof It will be enough to show that for $a(k, \varepsilon)$ sufficiently large,

$$
P(|V|=k,|E| \geqq a(k, \varepsilon)) \leqq \varepsilon .
$$

For any vertex $z \in S_{1}$, let $D(z)$ be the number of open edges from $z$ to $\mathbf{Z}^{d} \backslash S_{1}$ From Lemma 22 , it follows that for any $\varepsilon>0$, there exists an $L(\varepsilon)$ such that for any set $S \subseteq \mathbf{Z}^{d} \backslash S_{1}$, the conditional probability that $D(z)$ is at least $L(\varepsilon)$ given there is an open edge from $z$ to some element of $S$ is at most $\varepsilon$ We now let $a(k, \varepsilon)=k L(\varepsilon / k)$. 
The left-hand side in (2.2) is bounded from above by

$$
P\left(|V|=k, \sum_{z \in l} D(z) \geqq a(k, \varepsilon)\right)=\sum_{\substack{1 \leq s_{1} \\|\leq|=k}} P\left(\sum_{z \in A} D(z) \geqq a(k, \varepsilon) \mid V=A\right) P(V=A)
$$

It is therefore enough to show that for all sets $A \subseteq S_{1}$ of size $k, P\left(\sum_{z \in A} D(z) \geqq\right.$ $a(k, \varepsilon) \mid V=A)$ is at most $\&$ To prove this, we condition further on $S_{2}$ to obtain

$$
\begin{aligned}
& P\left(\sum_{z \in A} D(z) \geqq a(k, \varepsilon) \mid V=A\right) \\
& \quad=\sum_{I \subseteq \mathbf{Z}^{d} \backslash S_{!}} P\left(\sum_{z \in A} D(z) \geqq a(k, \varepsilon) \mid V=A, S_{2}=\Gamma\right) P\left(S_{2}=\Gamma \mid V=A\right),
\end{aligned}
$$

and so we now need only show that for any subset $\Gamma$ of $\mathbf{Z}^{d} \backslash S_{1}$ and any set $A \subseteq S_{1}$ of size $k$,

$$
P\left(\sum_{z \in A} D(z) \geqq a(k, \varepsilon) \mid V=A, S_{2}=\Gamma\right) \leqq \varepsilon
$$

However, conditioned on the event $\left\{V=A, S_{2}=\Gamma\right\}$ and using the measurability of $S_{2}$ with respect to the edges of $\mathbf{Z}^{d} \backslash S_{1}$, the random variables $\{D(z)\}_{z \in 1}$ are independent and by the way $L(\delta / k)$ was chosen, they each assume a value at least $L(\delta / k)$ with probability at most $\delta / k$ under the above conditioning Since

$$
\left\{\sum_{z \in A} D(z) \geqq k L(\delta / k)\right\} \subseteq \bigcup_{z \in A}\{D(z) \geqq L(\varepsilon / k)\},
$$

the conditional probability that $\sum_{z \in A} D(z) \geqq k L(\varepsilon / k)$ is at most $\varepsilon$, as desired

We now need to introduce some further notation. If $n \geqq m+1$, let $W_{m, n}$ be the set of vertices in $B(n-1)$ which are connected to $B(m)$ in the box $B(n-1)$ Let $F_{m, n}$ be the set of open edges from $W_{m, n}$ to $B(n-1)^{c}, E_{m, n}$ be the set of open edges from $W_{m . n}$ to $A^{\prime}(B(n-1))$ and $V_{m, n}$ be the set of vertices in $A^{l}(B(n-1))$ which have an open edge to $W_{m, n}$

Lemma 2.6. For all $k$ and $m \geqq 1$,

$$
\lim _{n \rightarrow \infty} P\left(\left|F_{m, n}\right| \leqq k, B(m) \rightarrow \infty\right)=0
$$

Proof Fix $k$ and $m$ Fatou's lemma tells us that

$$
\limsup _{n \rightarrow \infty} P\left(\left|F_{m, n}\right| \leqq k, B(m) \rightarrow \infty\right) \leqq P\left(\limsup _{n}\left\{\left|F_{m . n}\right| \leqq k, B(m) \rightarrow \infty\right\}\right)
$$

We now argue that the latter event has probability 0 Let $R_{n}$ be the set of vertices in $B(n-1)^{c}$ (possibly empty) which are the endpoint of an open edge in $F_{m, n}$ Define (random) integers $n_{1}, n_{2}$, inductively as follows Let $n_{1}=m+1, n_{2}=\inf \left\{i>n_{1}\right.$ $\left.R_{n_{1}} \subseteq B(i-1),\left|F_{m, i}\right| \leqq k\right\}$, and $n_{i+1}=\inf \left\{i>n_{l} \quad R_{n_{1}} \subseteq B(i-1),\left|F_{m, l}\right| \leqq k\right\}$ In addition, if $R_{n_{l}}=\emptyset$, let $n_{i+1}=\infty$ If some $n_{l}=\infty$, let all subsequent $n_{l}$ 's be $\infty$ 
Letting, for $r \geqq 1$,

$$
E_{i}=\left\{n_{1}<\infty, \text { there is an open edge between } R_{n_{1}} \text { and } W_{m n_{1}}^{\iota}\right\},
$$

it is immediate that

$$
\limsup _{n}\left\{\left|F_{m, n}\right| \leqq k, B(m) \rightarrow \infty\right\} \subseteq \bigcap_{l=1}^{\infty} E_{l},
$$

and so we need only show that the latter has probability 0 However, it is clear from independence and the fact that $\left|R_{n}\right| \leqq\left|F_{m, n}\right|$ that there exists $\alpha=\alpha(k)<1$ such that for all $r \geqq 1, P\left(E_{l+1} \mid E_{1} \cap E_{2} \cap \cap E_{l}\right) \leqq \alpha$ from which the above follows

Lemma 2.7. For all $k$ and $m \geqq 1$,

$$
\lim _{n \rightarrow \infty} P\left(\left|V_{m, n}\right| \leqq k, B(m) \rightarrow \infty\right)=0
$$

Proof Let $k, m \geqq 1$ and let $\varepsilon>0$ Let $c$ be as in $(21)$ and $a(k, \varepsilon)$ be as in Lemma 25 We then write

$$
\begin{aligned}
& P\left(\left|V_{m, n}\right| \leqq k, B(m) \rightarrow \infty\right) \leqq P\left(\left|V_{m, n}\right| \leqq k,\left|E_{m . n}\right| \geqq a(k, \varepsilon)\right) \\
& \quad+P\left(\left|E_{m . n}\right|<a(k, \varepsilon),\left|F_{m, n}\right| \geqq \frac{4}{c \varepsilon^{2}}+\frac{2 a(k, \varepsilon)}{c \varepsilon}\right) \\
& \quad+P\left(\left|F_{m . n}\right| \leqq \frac{4}{c \varepsilon^{2}}+\frac{2 a(k, \varepsilon)}{c \varepsilon}, B(m) \rightarrow \infty\right)
\end{aligned}
$$

The first term is at most $\&$ by Lemma 25 (with $S_{1}=\Lambda^{2}(B(n-1))$ and $\left.S_{2}=W_{m n}\right)$, the second term is at most $a$ by the second statement in Lemma 24 (with $R=\{z$ $d(\{z\}, B(n-1)) \geqq 2\}$ and $S=W_{m, n}$ and noting that $R \cup \Delta^{l}(R)=B(n-1)^{c}$ and $\left.\Delta^{l}(R)=\Delta^{l}(B(n-1))\right)$ and the third term goes to 0 as $n \rightarrow \infty$ by Lemma 26 . As $\varepsilon>0$ is arbitrary, the result follows

The next proposition is the analogue of Lemma 5 of [6] for long range percolation The main difficulty of extending their result to long range percolation is guaranteeing that there are many points on the vertex boundary of $B(n-1)$ which are connected in $B(n)$ to a smaller box $B(m)$ assuming that $\{B(m) \rightarrow \infty\}$. This latter fact was established by Lemma 27 We will prove Lemma 28 below to make this paper self contained but we mention that given Lemma 27 above, the proof essentially follows that of Lemma 5 in [6]

Lemma 2.8. Let $\alpha>0$ Then there exists integers $m$ and $n$ such that $2 m<n$ and

$$
P(B(m) \leftrightarrow K(m, n)(\text { in } B(n)))>1-\alpha
$$

Proof The first step of the proof is to show that for all $m$ and $\ell \geqq 1$,

$$
\liminf _{n} P\left(\left|V_{m . n}^{\prime}\right| \geqq l\right) \geqq 1-(P(B(m) \nrightarrow \infty))^{\frac{1}{d 2^{2 /}}}
$$

where $V_{m, n}^{\prime}$ is the set of vertices in $T(n)$ which have an open edge to $W_{m . n}$ To do this, we note that there exists a group of symmetries of the cube, of order 
$(2 d) 2^{d-1}=d 2^{d}$, and with the following property if the elements of the group transform $T(n)$ into $T_{1}(n), \quad, T_{d 2^{\prime \prime}}(n)$, then

$$
\Lambda^{l}(B(n-1)) \subseteq \bigcup_{l=1}^{d 2^{d}} T_{l}(n)
$$

It follows that

$$
\bigcap_{l=1}^{d 2^{d}}\left\{\left|V_{m, n}^{l}\right|<\ell\right\} \subseteq\left\{\left|V_{m, n}\right|<d 2^{d} \ell\right\},
$$

where $V_{m n n}^{l}$ is the set of vertices in $T_{l}(n)$ which have an open edge to $W_{m n}$ The FKG inequality gives us

$$
P\left(\left|V_{m, n}\right|<d 2^{d} \ell\right) \geqq \prod_{l=1}^{d 2^{d}} P\left(\left|V_{m, n}^{l}\right|<\ell\right)=\left(P\left(\left|V_{m, n}^{\prime}\right|<\ell\right)\right)^{d 2^{l}},
$$

which implies that

$$
P\left(\left|V_{m, n}^{\prime}\right| \geqq \ell\right) \geqq 1-P\left(\left|V_{m, n}\right|<d 2^{d} /\right)^{\frac{1}{d 2^{d}}}
$$

By Lemma 27 , it follows that

$$
\begin{aligned}
& \limsup _{n} P\left(\left\{\left|V_{m n}\right|<d 2^{d} /\right\}\right) \\
& \left.\quad=\limsup _{n} P\left(\left\{\left|V_{m, n}\right|<d 2^{d} /\right\} . B(m) \nrightarrow \infty\right) \leqq P(B(m) \nrightarrow \infty)\right)
\end{aligned}
$$

We therefore obtain

$$
\liminf _{n} P\left(\left|V_{m, n}^{\prime}\right| \geqq \ell\right) \geqq 1-(P(B(m) \nrightarrow \infty))^{\frac{1}{d 2^{d /}}},
$$

as desired

Next, since $\theta\left(p, p_{2}, p_{3}, \quad\right)>0$, there exists $m=m(d, p, x)$ so that

$$
P(B(m) \rightarrow \infty)>1-\left(\frac{1}{2} \alpha\right)^{d 2^{d}}
$$

Next let $q$ be the probability that $B(m)$ is an open $m$-pad and that there is an open edge between each vertex of $\left\{x \in B(m) x_{1}=-m\right\}$ and its unique nearest neighbor whose first coordinate is $-m-1$ Next choose $M$ sufficiently large so that at least one of $M$ independent events each with probability $q$ will occur with probability greater than $1-x / 2$

Next, choose $t$ so large that for any $n$ and for any subset $S$ of $T(n)$ of size $/$ or more, $S$ will have the property that there will be $M$ disjoint translates of $B(m)$ in $T(m, n)$ each distance 1 from some point of $S$ Next, by the first half of the proof, choose $n>2 m$ so large that

$$
P\left(\left|V_{m n}^{\prime}\right| \geqq \ell\right) \geqq 1-\frac{\%}{2}
$$

Finally, the way $t$ and $M$ were chosen, it follows that

$$
P(B(m) \leftrightarrow K(m, n)(\text { in } B(n)))>1-\varkappa
$$

The next step is to prove that there are paths from $A^{l}(R) \cap B(n)$ to $K(m, n)$ rather than just from $B(m)$ This is done in the next two lemmas 
Lemma 2.9. Let $\varepsilon>0$ and $l \geqq 1$ Then there exists $m$ and $n$ such that $2 m<n$ and such that if $B(m) \subseteq R \subseteq B(n)$ with $\left(R \cup A^{l}(R)\right) \cap T(n)=\emptyset$, then if $W$ is the set

$$
\left\{x \in\left(R \cup \Delta^{l}(R)\right)^{c} \cap B(n) \quad x \leftrightarrow K(m, n)\left(\text { in } B(n) \cap\left(R \cup \Delta^{l}(R)\right)^{c}\right)\right\}
$$

(which includes $K(m, n)$ by convention) and $F$ is the set of open edges from $(R \cup$ $\left.\Delta^{\prime}(R)\right) \cap B(n)$ to $W$, then $P(|F| \geqq \ell) \geqq 1-$ \&

Proof In the notation of Lemma 23 , we let $b=\sup _{l} p_{l}$ (where $\left.p_{1}=p\right), \gamma=\varepsilon / 2$ and $N=/$ and let $\delta$ be as in the conclusion of that lemma, requiring also that $\delta<\varepsilon / 2$ By Lemma 28 , we then choose integers $m$ and $n$ such that $2 m<n$ and

$$
P(B(m) \leftrightarrow K(m, n)(\text { in } B(n)))>1-\delta^{2}
$$

If we now have $R$ satisfying $B(m) \subseteq R \subseteq B(n)$ with $\left(R \cup \Delta^{l}(R)\right) \cap T(n)=\emptyset$, it follows that

$$
P\left(\left(R \cup \Delta^{l}(R)\right) \cap B(n) \leftrightarrow K(m, n)(\text { in } B(n))\right)>1-\delta^{2},
$$

which tells us that $P(|F| \geqq 1)>1-\delta^{2}$ It follows that there is a subset $\mathscr{P}^{\prime} \subseteq \mathscr{S}$ (where $\mathscr{S}$ is the set of all subsets of $\left.\left(R \cup \Delta^{l}(R)\right)^{c} \cap B(n)\right)$ such that $P(|F| \geqq 1 \mid W=$ $S) \geqq 1-\delta$ for all $S \in \mathscr{S}^{\prime}$ and $P\left(W \in \mathscr{S}^{\prime}\right) \geqq 1-\delta$ From Lemma 23 , the fact that $W$ is independent of the edges which have an endpoint in $\left(R \cup A^{r}(R)\right) \cap B(n)$, and the definition of $\delta$ it follows that if $S \in \mathscr{S}^{\prime}$, then $P(|F| \geqq \ell \mid W=S) \geqq 1-\delta / 2$ and hence $\left(\right.$ since $\left.P\left(W \in \mathscr{P}^{\prime}\right) \geqq 1-\delta\right) P(|F| \geqq \ell) \geqq(1-\varepsilon / 2)(1-\delta) \geqq 1-\varepsilon$, as desired

Lemma 2.10. Let $\&>0$ and $k \geqq 1$ Then there exists $m$ and $n$ such that $2 m<n$ and such that if $B(m) \subseteq R \subseteq B(n)$ with $\left(R \cup \Delta^{r}(R)\right) \cap T(n)=\emptyset$, then if

$$
V=\left\{x \in \Delta^{l}(R) \cap B(n) \quad x \leftrightarrow K(m, n)\left(\text { in } B(n) \cap\left(R \cup \Delta^{l}(R)\right)^{c}\right)\right\},
$$

then $P(|V|>k)>1-\varepsilon$

Proof. Let $a=a(k, \varepsilon / 2)$ be as in Lemma 25 Next choose $m$ and $n$ according to Lemma 29 with $\varepsilon$ replaced by $\varepsilon / 4$ and $\ell$ replaced by $\frac{4}{(\varepsilon ; 4)^{2}}+\frac{2 a}{(\varepsilon, 4)}$ Now let $R$ satisfy $B(m) \subseteq R \subseteq B(n)$ with $\left(R \cup A^{\prime}(R)\right) \cap T(n)=\emptyset$ Let $W$ be as in Lemma 29 . Then $V$ is the set of vertices in $\Delta^{\prime}(R) \cap B(n)$ which have an open edge to $W$ Let $E$ be the set of open edges from $A^{\prime}(R) \cap B(n)$ to $W$. By Lemma 25 (with $S_{1}=L^{\prime}(R) \cap B(n)$ and $\left.S_{2}=W\right)$,

$$
P(|V| \leqq k) \leqq P(|V| \leqq k,|E| \geqq a)+P(|E|<a)<\frac{\varepsilon}{2}+P(|E|<a),
$$

and so it suffices to show $P(|E|<a)<\frac{\varepsilon}{2}$ Letting $F$ be the set of open edges from $\left(R \cup \Delta^{l}(R)\right) \cap B(n)$ to $W$, we get

$$
\begin{aligned}
P(|E|<a) \leqq & P\left(|E|<a,|F| \geqq \frac{4}{c(\delta / 4)^{2}}+\frac{2 a}{c(\delta / 4)}\right) \\
& +P\left(|F|<\frac{4}{c(\delta / 4)^{2}}+\frac{2 a}{c(\varepsilon / 4)}\right)<\frac{\varepsilon}{4}+\frac{\varepsilon}{4},
\end{aligned}
$$

where the last inequalities follow respectively from the first statement in Lemma 2.4 (with $S=W$ ) and Lemma 29 together with the way $m$ and $n$ were chosen 
Proof of Lemma $A$ Choose $t$ such that $(1-\delta)^{t}<\varepsilon / 2$ Choose $m$ and $n$ according to Lemma 210 with $t$ and $\varepsilon / 2$ playing the role of $k$ and $\varepsilon$. Then if $R$ satisfies $B(m) \subseteq R \subseteq B(n)$ with $\left(R \cup A^{l}(R)\right) \cap T(n)=\emptyset, V$ is as in Lemma 210 , and $\beta$ $\Lambda^{e}(R) \cap B(n) \rightarrow[0,1-\delta]$, we have that $P(|V| \geqq t \mid H) \geqq 1-\varepsilon / 2$, since the random variable $|V|$ is independent of the event $H$. Next, each $v \in V$ is adjacent to some $\tilde{v} \in$ $R$ (if there is more than 1 , we choose such a $\tilde{v} \in R$ according to some predetermined nonrandom rule) Given $V$, let $\tilde{V}=\{\tilde{l} \quad v \in V\}$ By independence, the facts that $(1-\delta)^{t}<\delta / 2$ and $P(|V| \geqq t \mid H) \geqq 1-\varepsilon / 2$ give us

$$
P(|V| \geqq t,(v, \tilde{v}) \text { is }(\beta(v, \tilde{v})+\delta) \text {-open for some } v \in V \mid H) \geqq\left(1-\frac{\varepsilon}{2}\right)\left(1-\frac{\varepsilon}{2}\right)
$$

Finally, noting that

$$
\{|V| \geqq t,(v, \tilde{v}) \text { is }(\beta(v, \tilde{v})+\delta) \text {-open for some } v \in V\} \subseteq G
$$

completes the proof

\section{The Renormalization}

In this section we shall describe the renormalization anticipated earlier We shall see that Lemma A of the previous section implies that this renormalized model percolates and this in turn is easily seen to be enough to prove Theorem 11 The geometry of the renormalization is almost the same as that of [6], and as such we describe the procedure rather loosely referring to their paper for all missing details

Let $m$ and $n$ satisfy $2 m<n$ and set $N=n+m+1$. Let $d \geqq 2$ and order the edges between nearest neighbors of $\mathbf{Z}^{d}$ in some arbitrary way and consider boxes of the form $\left\{4 N z+B(N) \quad z \in \mathbf{Z}^{d}\right\}$ These boxes are called site boxes The translates of $B(N)$ which are exactly between two site boxes are called halfway boxes The idea is now to examine the site boxes one by one, declaring each of them to be either occupied or vacant according to certain rules to be specified The geometry of the procedure is as follows. We write $T_{j}(m, n)$ and $T_{j}(n)$ for the images of $T(m, n)$ and $T(n)$ respectively under the "earliest" isometries (in some arbitrary ordering) which preserve the origin and map the first coordinate direction onto the $j^{\text {th }}$, for $j=1, ., 2 d$ We define $K_{J}(m, n)$ as $K(m, n)$, replacing $T(m, n)$ and $T(n)$ by $T_{l}(m, n)$ and $T_{j}(n)$ respectively in the definition We start with the site box at the origin and call the site box $B(N)$ occupied if and only if the following (1)-(3) are all satisfied

(1) $B(m)$ is an open $m$-pad

(2) $B(m)$ is connected (in $B(n))$ to $K_{j}(m, n)$ for $j=1, \quad, 2 d$ The open $m$-pads in the $T_{j}(m, n)$ 's next to the $K_{,}(m, n)$ 's are called target open $m$-pads Note that for any $j$, there can of course be more than one such open target $m$-pad In such a case, we choose one according to a previously determined, nonrandom rule

(3) For all $j$, the target open $m$-pad in (2) in the $j^{\text {th }}$ direction (written as $b_{1}+B(m)$ ) is connected (in $b_{1}+B(n)$ ) to a vertex next (and connected) to an open $m$-pad in $b_{1}+T_{j}^{*}(m, n)$ in the halfway box next to $B(N)$ in the $j^{\text {th }}$ direction, where $T_{1}^{*}(m, n)$ is the image of $T_{j}(m, n)$ under the symmetry which fixes $e_{j}$ and sends, for $k \neq j$, the unit vector $e_{k}$ to $-e_{k}$ (We call this last $m$-pad again a target $m$-pad)

The reflections in (3) are necessary to make sure that the target open $m$-pads in (3) are indeed in the appropriate halfway box and the use of such reflections is 
called a steering action Note that the first open $m$-pad $B(m)$ is connected in two steps to target open $m$-pads in the halfway boxes. Next we consider the earliest edge starting at the origin in the ordering of the edges mentioned above, and we suppose without loss of generality that this edge is $e_{1}$, the unit vector in this direction We call the site box $B(N)+4 N e_{1}$ occupied if and only if the following $\left(1^{\prime}\right)$ and $\left(2^{\prime}\right)$ are satisfied

$\left(1^{\prime}\right)$ The target open $m$-pad in (3) above in the first direction is connected, in two steps which are analogues to the two steps described above, to a point next (and connected) to an open $m$-pad in $B(N)+4 N e_{1}$ Here we use an appropriate steering action to make sure that we end up in the correct site box

$\left(2^{\prime}\right)$ The target open $m$-pad in ( $\left.1^{\prime}\right)$ is in two steps connected to a point next (and connected) to an open $m$-pad in all halfway boxes next to $B(N)+4 N e_{1}$ which are also next to a site box which has not yet been examined (In this case there are $2 d-1$ such halfway boxes )

This procedure is continued Each time, we look for the "first" edge which connects some occupied site box to a site box which has not yet been examined and we decide whether or not the latter site box will be occupied or vacant Of course, we need the appropriate "steering action" each time to make sure that the target open $m$-pad ends up in the correct site box Again, details on this can be found in [6]

It is well known (see e $g$ [6]) that if at each step the conditional probability, given the past of the procedure, that a new site box is occupied is at least $q$ a s. where $q$ is independent of the site box being examined and strictly larger than the critical probability for independent nearest neighbor site percolation on $\mathbf{Z}^{d}$, then the origin of the renormalized (nearest neighbor) model percolates with positive probability The construction is such that the renormalized model percolating implies that the underlying long range model also percolates It is at this point where Lemma $\mathrm{A}$ comes in We shall now show that if $p \in(0,1)$ is such that $\theta\left(p, p_{2}, \quad\right)>0$, i e the underlying long range model percolates, and $\eta$ is a small positive number then the renormalized nearest neighbor model percolates under $p+\eta$ for a suitable choice of $n$ and $m$ (Here and in what follows, percolation under a certain parameter $q$ refers to the fact that the probability for an edge of length one to be open is q.)

Fix $p$ so that $\theta\left(p, p_{2}, \quad\right)>0$ and let $\eta>0$ Let

$$
\delta=\frac{\eta}{2 d}
$$

and

$$
\varepsilon=\frac{\left(1-p_{c}(\text { site })\right)}{8 d}
$$

where $p_{c}$ (site) denotes the critical probability for independent nearest neighbor site percolation on $\mathbf{Z}^{d}$ Let $m$ and $n$ be as in Lemma A for this choice of $p, s$ and $\delta$ We shall explain why the renormalized model with this choice of $m$ and $n$ percolates for $p+\eta$

First of all, the probability that $B(m)$ is an open $m$-pad under $p$ is positive It doesn't matter that this probability is in fact very small, it serves only as a starting point for the procedure For inductive reasons, we write $C_{1}$ for the set of open edges in $B(m)$, where open is to be interpreted as $p$-open if the edge has length one To estimate the probability under $p$ that the event described in (2) above occurs, note 
that given the fact that $B(m)$ is an open $m$-pad under $p$, we have no information about edges in $A^{e}(B(m))$ and so all we can say is that they are 0-closed Now Lemma A implies that with probability at least $1-\varepsilon$ there is a path from $B(m)$ to $K(m, n)$ via $\Delta^{c}\left(B(m)\right.$ ) (in $B(n)$ ) such that the edge in $\Delta^{e}(B(m))$ is $\dot{\delta}$-open and all other edges are open (meaning $p$-open if the edge has length one) By symmetry it follows that the event in (2) occurs under $p$ with probability at least $1-2 d \varepsilon$ Now define

$$
C_{2}=C_{1} \cup E_{1} \cup F_{1},
$$

where $E_{1}$ is the set of edges in $A^{e}(B(m))$ which are $\delta$-open and $F_{1}$ is the set of open edges $\{x, y\}$ contained in $B(n) \cup \bigcup_{j=1}^{2 d} T_{j}(m, n) \backslash B(m)$ which are open ( $p$ open for edges of length one) and such that there is an open path in $B(n) \cup$ $\bigcup_{j=1}^{2 d} T_{l}(m, n) \backslash B(m)$ from $x$ (and hence from $y$ ) to some point in $\Delta^{\prime}(B(m)$ ) which is an endpoint of some edge in $E_{1}$ Assuming that the event in (2) above occurs, we have the following information about the state of the edges outside $C_{2}$ which share at least one endpoint with an edge of $C_{2}$ All edges in $\Delta^{e}(B(m)) \backslash C_{2}$ are $\delta$-closed, and all other edges outside $C_{2}$ which share at least one endpoint with an edge in $C_{2}$ and which are contained in $B(n) \cup \bigcup_{j=1}^{2 d} T_{,}(m, n) \backslash B(m)$ are closed (meaning $p$-closed if the edge has length one) We have no information about any other edges Next we estimate the probability under $p+\eta$ that all events in (3) above occur, given the information just described For this we apply Lemma A $2 d$ times, this time "centered" at $b$, rather than at the origin Let $R$ be the set of endpoints of edges in $C_{2}$ inside $b_{1}+B(n)$ For each edge $e$ in $\Lambda^{e}(R)$ we know that $e$ is $\beta(e)$-closed, where $\beta(e)$ is either $0, \delta$ or $p$ The extra information about closed edges of length at least two is irrelevant for the occurrence of the event $G$ in Lemma A and will also be irrelevant in the future of the procedure Hence we can apply Lemma A as before and conclude that the (conditional) probability under $p+\delta$ of the first of the $2 d$ events in (3) is at least $1-8$ We repeat this procedure for the remaining events in (3), noting that any particular edge is "updated" at most $2 d+1$ times (We shall see that this will be true for all edges in the future of the procedure ) This update is by an amount $\delta$ at most $2 d$ times and by an amount $p$ at most once Hence the open cluster we find is open under $p+2 d \delta=(p+\eta)$ The probability that the site box at the origin is occupied under $p+\eta$, given that $B(m)$ is $p$-open is at least $(1-2 d \varepsilon)(1-\varepsilon)^{2 d}$ It is easy to check that by our choice of $\varepsilon$ this probability is at least $\frac{1}{2}\left(1+p_{c}\right.$ (site $\left.)\right)$ which is strictly larger than $p_{c}$ (site)

It remains to show that also the events described in $\left(1^{\prime}\right)$ and $\left(2^{\prime}\right)$ have sufficiently high probability under $p+\eta$ In principle this can be shown in the same way as above We must be careful however, because it is very well possible that a certain site box was declared to be vacant and this is negative information which can be used against us However, the construction is such that any new target $m$-pad is always unconditioned In fact, this is the reason that we need the halfway boxes if we tile the space with translated copies of $B(N)$ and try to connect them up directly without using halfway boxes, then the event that a certain site box is vacant has the effect that a potential future open $m$-pad is less likely to be open Finally, we observe that all $r$-open edges of length one which we use in the construction above satisfy $r<p+\eta$ We have shown the following

Theorem 3.1. Consider a long range model $p, p_{2}, p_{3}$, on $\mathbf{Z}^{d}, d \geqq 2$ satisfying Condition $C$ and suppose that $p \in(0,1)$ and $0\left(p, p_{2}, p_{3}, \quad\right)>0$ For any, 
$\eta>0$ there exist $m$ and $n$ such that the renormalized site percolation model with underlying long range model $p+\eta, p_{2}, p_{3}$, . . described above percolates as a nearest neighbor model

Proof of Theorem 11 For all $n$ we have $p_{c}\left(p_{2}^{n}, p_{3}^{n}, \quad\right) \geqq p_{c}\left(p_{2}, p_{3}\right.$, . $)$ We may assume that $p_{c}\left(p_{2}, p_{3}, \quad\right)<1$ Let, for all $\alpha>0, p^{(\varkappa)}$ be defined by $p^{(\varkappa)}=$ $p_{c}\left(p_{2}, p_{3}, \quad\right)+\alpha$ For any $s>0$, we have that $0\left(p^{(i, 2)}, p_{2}, p_{3}, \quad\right)>0$, and it then follows from Theorem 31 that for a suitable choice of the parameters, the renormalized model based on the long range model $p^{(i)}, p_{2}, p_{3}$, percolates The key observation is that the event that a certain site box is occupied depends on the state of a uniformly bounded number of edges which are all of $L_{1}$-length at most $4 N$, where $N$ is as in the construction of the renormalization We recall that the reason that the renormalized model percolates is that the conditional probability that a new site box is occupied, conditioned on the past of the procedure, is at least $q$ a $s$ for some uniform $q>p_{c}$ (site) Note that in all applications of Lemma A, there can be only finitely many different events we condition on It then follows from the pointwise convergence of the models $p^{(i)}, p_{2}^{n}, p_{3}^{n}$, . and the key observation above that for $n$ sufficiently large, the conditional probability that a new site box is occupied in the renormalization based on $p^{(i)}, p_{2}^{n}, p_{3}^{n}$, is at least $q^{\prime}$ for some $q^{\prime}>p_{c}$ (site) It follows that for $n$ large enough, $p_{c}\left(p_{2}^{n}, p_{3}^{n}, \quad\right) \leqq p_{c}\left(p_{2}, p_{3},.\right)+\varepsilon$ and the proof is complete since $\varepsilon>0$ was arbitrary

\section{Further Proofs and Applications}

The first part of this section is devoted to a proof of Theorem 14 We start with a lemma which is stronger than we actually need A part of the argument of this lemma is a long range version of the argument in [3] for proving continuity from the left of the percolation function in independent nearest neighbor percolation in the supercritical regime

Lemma 4.1. Let $p_{2}, p_{3}$, be a long range model and assume that we have a sequence of long range models $\left(p_{2}^{n}, p_{3}^{n}\right.$. . ) such that $p_{1}^{n} \leqq p_{i}$ for all $n \geqq 1$ and $i \geqq 2$ and $\lim _{n \rightarrow \infty} p_{t}^{n}=p_{i}$ for all $i \geqq 2$ Then the following two statements (i) and (ii) are equivalent

(i) If $p_{1}>p_{1}\left(p_{2}, p_{3}, \quad\right)$ and $p_{1}^{n} \rightarrow p_{1}$ from below, then

$$
\lim _{n \rightarrow \infty} O\left(p_{1}^{n}, p_{2}^{n}, \quad\right)=0\left(p_{1}, p_{2}, \quad\right)
$$

$$
\lim _{n \rightarrow \infty} p_{c}\left(p_{2}^{n}, p_{3}^{n}, \quad\right)=p_{c}\left(p_{2}, p_{3}, \quad\right)
$$

Proof First we prove that (i) implies (ii) We clearly have that for all $n \geqq 1$,

$$
p_{c}\left(p_{2}^{n}, p_{3}^{n}, \quad\right) \geqq p_{c}\left(p_{2}, p_{3}, \quad\right),
$$

and so to prove (ii), it suffices to show that if $p_{1}>p_{c}\left(p_{2}, p_{3}, \quad\right)$, then $p_{1} \geqq$ $p_{c}\left(p_{2}^{n}, p_{3}^{n}, \quad\right)$ for $n$ sufficiently large Now, letting $p_{1}^{n}=p_{1}$ for all $n \geqq 1$, it follows from (i) that

$$
\theta\left(p_{1}, p_{2}^{n}, p_{3}^{n}, \quad\right)=0\left(p_{1}^{n}, p_{2}^{n}, p_{3}^{n}, \quad\right) \rightarrow 0\left(p_{1}, p_{2}, \quad\right)>0,
$$


and hence $\left(p_{1}, p_{2}^{n}, p_{3}^{n}, \quad\right)>0$ for $n$ sufficiently large, whence

$$
p_{1} \geqq p_{c}\left(p_{2}^{n}, p_{3}^{n}, \quad\right)
$$

for all $n$ large enough

Next we show that (ii) implies (i) We fix $p_{1}>p_{c}\left(p_{2}, p_{3}, \quad\right)$, and a sequence $\left(p_{1}^{\prime \prime}\right)$ converging to $p_{1}$ from below It suffices to show that

$$
\theta\left(p_{1}, p_{2}, \quad\right) \leqq \liminf _{n \rightarrow \infty} \theta\left(p_{1}^{n}, p_{2}^{n}, .\right)
$$

Using (ii), we have that for $n$ large enough, $p_{1}^{n}>p_{c}\left(p_{2}^{n}, p_{3}^{n}\right.$, ) We can couple all models $\left(p_{1}, p_{2}, \quad\right)$ and $\left(p_{1}^{n}, p_{2}^{n}, \quad\right)$ on the same probability space similar to the discussion preceding Lemma A for any two vertices $x$ and $y$ of $\mathbf{Z}^{d}$, the edge $\{x, v\}$ is associated with a uniform- $(0,1)$ distributed random variable $(1)(x, 1)$ which is independent of all other such random variables. The edge $\{x, y\}$ is declared to be open in $\left(p_{1}^{n}, p_{2}^{n}, \quad\right)$ iff $(1)(x, y)<p_{1, y^{n}}^{n}$, and similarly, $\left\{x, y^{\prime}\right\}$ is open in $\left(p_{1}, p_{2}, \quad\right)$ iff $(1)(x, 1)<p_{|1-1|}$

The model $p_{1}, p_{2}$, is supercritical by assumption and hence there is as a unique infinite cluster, (see [4]) For all $n$ sufficiently large, the model $\left(p_{1}^{n}, p_{2}^{n}, \quad\right.$ ) is also supercritical and contains therefore (again by [4]) a unique infinite cluster a s In the coupling of the models described above, the unique infinite cluster in these models has to be contained in the unique infinite cluster corresponding to $p_{1}, p_{2}$,

Now suppose that the origin percolates in $p_{1}, p_{2},$. , and denote this event by $\left\{0 \rightarrow \infty\right.$ in $\left.p_{1}, p_{2}, \quad\right\}$ The infinite cluster in $p_{1}^{n}, p_{2}^{n}$, need not contain the origin, but there must be a finite path from the origin to this cluster which is contained in the infinite cluster of $p_{1}, p_{2}$, Each edge $\{x, y\}$ along this finite path has (') $(1, v)<p_{\mid,-1\}}$ (note the strict inequality) and since $p_{l}^{n} \rightarrow p_{l}$ for all $i$, we have shown that if the origin percolates in $p_{1}, p_{2}$, , then it also percolates in $p_{1}^{n}, p_{2}^{n}$, for all $n$ sufficiently large Writing $\left\{0 \rightarrow \infty\right.$ in $\left.p_{1}^{n}, p_{2}^{n}, \quad\right\}$ for these last events, we have shown that in the coupling described above,

$$
\left\{0 \rightarrow x \text { in } p_{1}, p_{2}, .\right\} \subseteq \liminf _{n \rightarrow \infty}\left\{0 \rightarrow x \text { in } p_{1}^{n}, p_{2}^{n}, \quad\right\},
$$

and the result follows from an application of Fatou's lemma

In the next lemma we consider approximation from above This result is a long range version of the well known right continuity of the percolation function for ordinary percolation

Lemma 4.2. Consider the models $p_{1}, p_{2}$, and $p_{1}^{n}, p_{2}^{n}$, for $n \geqq 1$ and liew them as functions from $\mathbf{Z}^{d}$ into $[0,1]$ Suppose that $p_{i}^{n} \geqq p_{i}$ for all $i$ and $n$, and suppose that $p_{1}^{n}, p_{2}^{n}$, converges to $p_{1}, p_{2}$, in $L_{1}$ of $\mathbf{Z}^{d}$ with counting measure Then $\lim _{n \rightarrow \infty} \theta\left(p_{1}^{n}, p_{2}^{n}, \quad\right)=0\left(p_{1}, p_{2}, \quad\right)$

Proof The assumption on the $L_{1}$-convergence implies that

$$
\lim _{m \rightarrow \infty} \sup _{n} \sum_{z \in B(m)^{r}} p^{n}(z)=0
$$

In particular, the probability that there is an open edge from the origin to $B(m)^{c}$ tends to zero uniformly in $n$ when $m \rightarrow \propto$ We write $P_{p}$ for the probability measure 
corresponding to $p_{1}, p_{2}$, and $P_{p^{n}}$ for the probability measure corresponding to $p_{1}^{n}, p_{2}^{n}, \quad$ Fix $\varepsilon>0$ and take $k$ so large that

$$
P_{p}\left(0 \leftrightarrow B(k)^{c}\right)<0\left(p_{1}, p_{2}, \quad\right)+\varepsilon / 3
$$

Next choose $m$ so large that the probability that there is an edge with one endpoint in $B(k)$ and the other endpoint in $B(m)^{c}$ is smaller than $\varepsilon / 3$, uniformly in all models (This is possible since the probability of this event is bounded from above by $(2 k+$ $1)^{d}$ times the probability that there is a direct connection from 0 to $\left.B(m-k)^{c}\right)$ We now write

$$
\begin{aligned}
O\left(p_{1}^{\prime \prime}, p_{2}^{n}, \quad\right) & =P_{p^{\prime \prime}}(0 \rightarrow \infty) \\
& \leqq P_{p^{\prime \prime}}(0 \leftrightarrow B(m) \backslash B(k) \text { in } B(m))+P_{p^{\prime \prime}}\left(\exists \text { edge from } B(k) \text { to } B(m)^{c}\right) \\
& \leqq P_{p^{n}}(0 \leftrightarrow B(m) \backslash B(k) \text { in } B(m))+c / 3
\end{aligned}
$$

Note that the first event in the last line depends only on edges in $B(m)$ and by the pointwise convergence of the model we can take $n$ so large that

$$
P_{p^{\prime \prime}}(0 \leftrightarrow B(m) \backslash B(k) \text { in } B(m)) \leqq P_{p}(0 \leftrightarrow B(m) \backslash B(k) \text { in } B(m))+\varepsilon / 3
$$

It follows that

$$
\begin{aligned}
P_{p^{n}}(0 \leftrightarrow B(m) \backslash B(k) \text { in } B(m)) & \leqq P_{p}\left(0 \leftrightarrow B(k)^{\varsigma}\right)+\varepsilon / 3 \\
& \leqq 0\left(p_{1}, p_{2}, \quad\right)+2 \varepsilon / 3
\end{aligned}
$$

Putting things together we find that for $n$ sufficiently large,

$$
\theta\left(p_{1}^{\prime \prime}, p_{2}^{\prime \prime}, \quad\right) \leqq 0\left(p_{1}, p_{2}, \quad\right)+\varepsilon,
$$

and the proof is complete, noting that $\theta\left(p_{1}^{n}, p_{2}^{n}, \quad\right) \geqq 0\left(p_{1}, p_{2}, \quad\right)$ for all $n \quad \square$

Proof of Theorem 14 The first statement of Theorem 14 is Lemma 42 For the second statement, we assume that $p_{2}, p_{3}$, satisfies Condition $\mathrm{C}$ and that $p_{1}>p_{c}\left(p_{2}, p_{3}, \quad\right)$ If $p_{l}^{n} \leqq p_{l}$ for all $n \geqq 1$ and $i \geqq 2$, then we have from Theorem 11 that $\lim _{n \rightarrow \infty} p_{c}\left(p_{2}^{n}, p_{3}^{n}, \quad\right)=p_{c}\left(p_{2}, p_{3}, \quad\right)$ If $p_{1}^{n} \rightarrow p_{1}$ from below, it follows from Lemma 41 that ()$\left(p_{1}^{n}, p_{2}^{n}, \quad\right) \rightarrow O\left(p_{1}, p_{2}, \ldots\right)$ We thus have the required convergence if the approximating models are such that $p_{i}^{\prime \prime} \leqq p_{i}$ for all $n \geqq 1$ and all $i \geqq 1$ To complete the proof, given $p_{1}^{n}, p_{2}^{n}$, which converges to $p_{1}, p_{2}$, (in $L_{1}$ of $\mathbf{Z}^{d}$ with counting measure) we "sandwich" the approximating models between two other models, one of which is above the limit and one of which is below More precisely, let $Q_{l}^{n}=\max \left\{p_{l}^{n}, p_{l}\right\}$ and $q_{l}^{n}=\min \left\{p_{l}^{n}, p_{l}\right\}$ for all $i \geqq 1$ and $n \geqq 1$ A simple coupling argument shows that $\theta\left(q_{1}^{n}, q_{2}^{n}, \quad\right) \leqq 0\left(p_{1}^{n}, p_{2}^{n}, \quad\right) \leqq$ $\theta\left(Q_{1}^{n}, Q_{2}^{n}, \quad\right)$. Since $p_{1}^{n}, p_{2}^{n}$, converges in $L_{1}$ to $p_{1}, p_{2}$, , it follows that both $Q_{1}^{n}, Q_{2}^{n}, \quad$ and $q_{1}^{n}, q_{2}^{n}, \quad$ converge to $p_{1}, p_{2}, \quad$ in $L_{1}$ and the proof is complete

Lemma $\mathrm{A}$ and the renormalization technique have further consequences Section 5 in [6] contains some of these in the case of independent nearest neighbor percolation on $\mathbf{Z}^{d}$. Here, we give some analogues of their results for long range percolation models Let $F \subseteq \mathbf{Z}^{d}$ be an infinite set which is connected in the usual 
nearest neighbor structure of $\mathbf{Z}^{d}$ The critical probability for independent nearest neighbor site percolation on $F$ is denoted by $p_{c}^{F}$, and the critical probability for the long range model $p_{2}, p_{3}$, on $F$, defined in the obvious way, is denoted by $p_{c}^{F}\left(p_{2}, p_{3}, \quad\right)$

Theorem 4.3. Let $F \subseteq \mathbf{Z}^{d}$ be an infinite set, connected in the usual nearest neighbor structure such that $p_{c}^{F}<1$ For any long range model $p_{2}, p_{3}$, which satisfies Condition $\mathrm{C}$ we have

$$
\lim _{h \rightarrow \infty} p_{c}^{2 h F+B(k)}\left(p_{2}, p_{3}, \quad\right)=p_{c}\left(p_{2}, p_{3}, \quad .\right)
$$

If we take $F$ to be the half space $\mathbf{H}^{d}=\left\{z \in \mathbf{Z}^{d} \quad z_{1} \geqq 0\right\}$ for any $d \geqq 2$, or the slice $S(k)=\left\{z \in \mathbf{Z}^{d} \quad 0 \leqq z_{j} \leqq k, j>2\right\}$ for any $d \geqq 3$, we immediately obtain the following corollary

Corollary 4.4. For $d \geqq 2$ and any long range model $p_{2}, p_{3}$, . which satisfies Condition $\mathrm{C}$, we have

$$
p_{c}^{\mathbf{H}^{\prime}}\left(p_{2}, p_{3}, \quad\right)=p_{c}\left(p_{2}, p_{3}, \quad\right) .
$$

For $d \geqq 3$ and any, long range model $p_{2}, p_{3}$, which satisfies Condition $\mathrm{C}$ we have

$$
\lim _{h \rightarrow \infty} p_{c}^{S(h)}\left(p_{2}, p_{3}, \quad\right)=p_{c}\left(p_{2}, p_{3}, \quad\right)
$$

Proof of Theorem 43 In the renormalization, we consider site boxes one by one according to a certain order of the edges in $\mathbf{Z}^{d}$ The only thing that needs to be done now is to order the edges in $F$ rather than in $\mathbf{Z}^{d}$ and go from site box to site box according to this order in the manner we did earlier The fact that $p_{c}^{F}<1$ guarantees that if a long range model satisfying Condition $\mathrm{C}$ is supercritical, then we can take the boxes in the renormalization so large that the renormalized model also percolates (as a nearest neighbor site percolation model). In other words, the long range percolation model percolates in $2 k F+B(k)$ for $k$ sufficiently large (see [6] for more details).

\section{Proof of Theorem 1.3}

In this section, we give the proof of Theorem 13

Proof of Theorem 13 Case (i) is trivial and left to the reader. We prove case (ii) for $d \geqq 2$ by induction on $d$ (with $p_{2}, p_{3}$. . fixed) Let $p_{1} \in(0,1)$ For each $d \geqq 2$, we will prove the existence of a constant $c_{d}>0$ (depending on $p_{1}$ ) so that in $d$ dimensions if $R \subseteq B(n), \hat{\imath} \in B(n)$ and $\imath \notin R \cup \Delta^{r}(R)$, then

$$
\sum_{x \in A^{\prime}(R) \cap B(n)} p\left(x-\vartheta^{\prime}\right) \geqq c_{d} \sum_{x \in\left(R \cup d^{\prime}(R)\right) \cap B(n)} p\left(x-\vartheta^{\prime}\right)
$$

We first prove this for $d=2$ We first claim that there exists a constant $\kappa>0$ such that for all $n \geqq 1$,

$$
p_{n} \geqq \kappa \sum_{1 \cdot \gamma_{2} \geqq n} p(x)
$$


It is easy to see that we need only find $\kappa>0$ so that $(51)$ holds for large $n$ However, if $n \geqq N_{0}$, we have

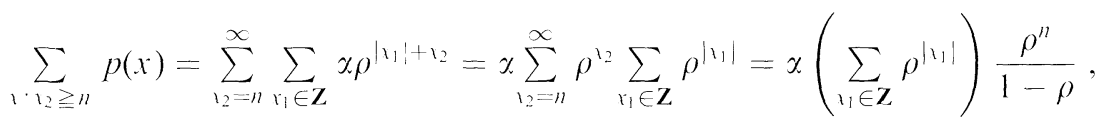

from which the existence of $\kappa$ follows

A similar computation shows that the above $k$ can also be taken sufficiently small so that for all $\left(x_{1}, x_{2}\right) \neq(0,0)$ with $x_{1} \geqq 0$,

$$
p\left(\left(x_{1}, x_{2}\right)\right) \geqq \kappa \sum_{\left(a, 1_{2}\right), a \geqq r_{1}} p\left(\left(a, x_{2}\right)\right)
$$

We will now for simplicity assume that $\hat{\gamma}=0$, the more general case being proved analogously

Let

$$
t=\min \left\{m>0 .(0, m) \in R \cup \Delta^{r}(R)\right\}
$$

and

$$
b=\max \left\{m<0 \cdot(0, m) \in R \cup A^{r}(R)\right\},
$$

which may be $\propto$ or $-\infty$ respectively Equation (5 1) now gives us that

$$
p((0, t)) \geqq \kappa \sum_{i \in R \cup A^{\prime}(R): r_{2} \geqq t} p(x)
$$

and

$$
p((0, b)) \geqq \kappa \sum_{1 \in R \cup \Delta^{i}(R) \cdot x_{2} \leqq b} p(x)
$$

To exhaust all points in $R \cup A^{r}(R)$, we next take an integer $j \in(b, t)$ (noting that $\left.(0, j) \notin R \cup \Delta^{\prime}(R)\right)$ and consider

$$
r_{j}=\min \left\{m>0 \quad(m, j) \in R \cup \Delta^{l}(R)\right\}
$$

and

$$
\ell_{1}=\max \left\{m<0 \quad(m, j) \in R \cup \Delta^{l}(R)\right\},
$$

which again may be $\infty$ or $-\infty$ respectively. Equation (52) (together with its reflected version) gives us that

$$
p\left(r_{1}, j\right) \geqq \kappa \sum_{(a, j) \in R \cup A^{\prime}(R)} p((a, j))
$$

and

$$
p\left(t_{j}, j\right) \geqq k \sum_{(a, j) \in R \cup A^{\prime}(R) . a \leqq \prime,} p((a, j))
$$

Of course, if $t, b$ or some $r$, or $t$, is $\pm \infty$, the relevant sum does not appear Next, the union of the right-hand sides in (5 3), (5 4), (5 5) and (5 6) (with $j$ varying over $(b, t))$ is $R \cup A^{\prime}(R)$ and the left-hand sides in the same equations all correspond to different points in $A^{\prime \prime}(R) \cap B(n)$ This proves the result for $d=2$ with $c_{2}=k$

For $d \geqq 3$, we perform induction as follows Assume now that for $i=2$, , $d-1$, there is a constant $c_{l}>0$, depending on $p_{1}$ such that in $i$ dimensions if 
$R \subseteq B(n), ; \in B(n)$ and $\hat{\imath} \notin R \cup \Lambda^{l}(R)$, then

$$
\sum_{r \in N^{\prime}(R) \cap B(n)} p(x-\hat{\imath}) \geqq c_{l} \sum_{i \in\left(R \cup A^{\prime}(R)\right) \cap B(n)} p\left(x-\hat{\imath}^{\prime}\right)
$$

Next note that $(51)$ can easily be generalized to give us a constant $k_{d}$ (depending only on the dimension) such that for all $n \geq 1$,

$$
p_{n} \geqq \kappa_{d} \sum_{r \cdot v_{d} \geqq n} p(x)
$$

As we did for $d=2$, we let

$$
t=\min \left\{m>0 \quad(0, \quad, 0, m) \in R \cup \Delta^{l}(R)\right\}
$$

and

$$
b=\max \left\{m<0 \quad(0, \quad, 0, m) \in R \cup \Delta^{l}(R)\right\},
$$

which may again be $\infty$ or $-\infty$ respectively Equation (57) now gives that

$$
p(0, \quad, 0, t) \geqq \kappa_{d} \sum_{i \in R \cup l^{\prime}(R), l_{d} \geqq t} p(x)
$$

and that

$$
p(0, \quad, 0, b) \geqq \kappa_{d} \sum_{1 \in R \cup 1^{\prime}(R) \cdot \gamma_{d} \leqq h} p(r)
$$

Now, for integers $j \in(b, t)$, consider the hyperplane

$$
\mathscr{H}_{j}=\left\{x \quad x_{d}=j\right\}
$$

Let $R_{J}=R \cap \mathscr{H}$, and view this (with a slight abuse of notation) as a subset of $\mathbf{Z}^{d-1}$ Letting $\Lambda^{\prime}\left(R_{J}\right)=\left\{x \in \mathscr{H}_{,} \backslash R_{J},|x-y|=1\right.$ for some $\left.y \in R_{J}\right\}$ (which we think of as the vertex boundary of $R$, within the hyperplane $\left.\mathscr{H}_{j}\right)$, we have that $\Delta^{\prime}\left(R_{J}\right) \subseteq \Delta^{l}(R) \cap$ $\mathscr{H}_{1}$ (with possible proper containment) and so, by the induction assumption,

$$
\sum_{i \in I^{\prime}\left(R_{,}\right) \cap B(n)} p((0, \quad, 0, j)-x) \geqq \mathcal{C}_{d-1} \sum_{i \in\left(R, \cup d^{\prime}\left(R_{,}\right)\right) \cap B(n)} p((0, \quad ., 0, j)-x)
$$

Next, the conditions of our $p_{n}$ 's clearly imply the existence of two positive constants $C^{\prime}$ and $C^{\prime \prime}$ so that for all positive integers $u$ and $v$, we have that

$$
p_{u+\imath} \geqq C^{\prime} \rho^{u} p_{\imath} \quad \text { and } \quad p_{u+\imath} \leqq C^{\prime \prime} \rho^{u \prime} p_{\imath}
$$

This together with (5 10) implies that

$$
\sum_{l \in 1^{\prime}(R,) \cap B(n)} p(x) \geqq \frac{c_{d-1} C^{\prime}}{C^{\prime \prime}} \sum_{1 \in\left(R, \cup d^{\prime}(R,)\right) \cap B(n)} p(x)
$$

As before, the right-hand sides of (5 8), (59) and (5 11) exhaust $\left(R \cup A^{2}(R)\right) \cap B(n)$ and the left-hand sides correspond to different points in $\Lambda^{\prime}(R) \cap B(n)$ Letting $c_{d}=$ $\min \left\{\kappa_{d}, \frac{C_{d-1} C^{\prime}}{C^{\prime \prime}}\right\}$, the result is proved

Acknowledgement We thank Karm Nelander for a number of corrections and comments on the first version of this paper 


\section{References}

1 Aizenman, M and Grimmett, G R : Strict Monotonicity for Critical Ponts in Percolation and Fer1omagnetic Models J Stat Phy 63, 817-835 (1991)

2 Aizenman, $M$ and Newman, $C$ : Discontinuity of the percolation density in the onedimensional 1/i.x $-\left.1\right|^{2}$ percolation models Commun Math Phys 107, 611-647 (1986)

3 Beig, J van den and Keane, $M$ : On the continuity of the percolation probability function In: R Beals et al, (ed) Conference on Modern Analysis and Probability Contemporary Mathematics 26, Providence, Rhode Island; American Mathematical Society, pp 61-65 (1984)

4 Gandolfi, A, Keane, $M$ and Newman, $C$ : Uniqueness of the infinite component in a 1 andom graph with applications to percolation and spin glasses Prob Th Rel Fields 92, 511-527 (1992)

5 Giimmett, G: Peıcolation Berlin-Heidelberg-New York Springer-Verlag (1989)

6 Gi immett, G R and Marstrand, JM: The supercritical phase of percolation is well behaved Proceedings of the Royal Society of London A 430, 439-457 (1990)

7 Haris, I E A lower bound for the critical probability in a certan percolation process Proc Cambridge Phil Soc 56, 13-20 (1960)

8 Kesten, $\mathrm{H}$. The critical probability of bond percolation on the square lattice equals $\frac{1}{2} \mathrm{Com}$ mun Math Phys 74, 41-59 (1980)

9 Newman, C and Schulman, LS: One-dimensional $1 /|r-1|^{\prime}$ petcolation models Commun Math Phys 104, 547-571 (1986)

Communicated by A Jaffe 